https://doi.org/10.15407/ujpe64.7.602

O. KOVALENKO

National Centre for Nuclear Research

(Warsaw, Poland; e-mail: oleksandr.kovalenko@ncbj.gov.pl)

\title{
NEUTRAL MESON AND DIRECT PHOTON MEASUREMENTS WITH THE ALICE EXPERIMENT
}

\begin{abstract}
The ALICE experiment is designed to study the properties of the matter created in protonproton and heavy-ion collisions at the LHC. Neutral mesons can be reconstructed in ALICE in a wide range of transverse momenta via two-photon decays. Neutral meson measurements in $p p$ collisions give an opportunity to validate the NLO or NNLO $p Q C D$ calculations and to constrain the parton distribution functions and the parton fragmentation functions. Neutral meson spectra measured in $p A$ and $A A$ collisions allow us to test a modification of the parton distribution functions in nuclei and the parton energy loss in the hot matter created in $A A$ collisions. The recent results from ALICE on direct photon measurements in the $\mathrm{Pb}-\mathrm{Pb}$, neutral pion and $\eta$ meson productions in $p p, p-P b$, and $P b-P b$ collisions are presented.

Ke ywords: high-energy physics, neutral meson spectra, direct photons.
\end{abstract}

\section{Introduction}

ALICE experiment aims to explore properties of the hot $\left(T \sim 10^{12} \mathrm{~K}\right)$ and dense quark-gluon matter and to investigate the chiral symmetry restoration and the deconfinement mechanisms. The hard hadron production in pp collisions can be described by a convolution of the hard parton cross-section, parton distribution function (PDF), and fragmentation function $(\mathrm{FF})$. The measurement of hadron spectra in a wide kinematic range for various collision energies provides a new input for PDF and FF parametrizations. The meson production in heavy-ion collisions allows studying several effects. The development of a collective flow can be studied at low $p_{\mathrm{T}}\left(p_{\mathrm{T}}<\right.$ $<3 \mathrm{GeV} / c)$. The high- $p_{\mathrm{T}}\left(p_{\mathrm{T}}>5 \mathrm{GeV} / c\right)$ part of the spectra originates predominantly from the hard parton hadronization. At moderate $p_{\mathrm{T}}$, the $\pi^{0}$ and $\eta$ mesons are mainly produced via the gluon fragmentation at LHC energies. As gluons show a larger energy loss in the medium than quarks, the comparison of the suppressions of the yields of light neutral mesons and heavier hadrons will provide an input for the understanding of the energy loss by the different partons. The difference in the suppression patterns of the $\pi^{0}$ and $\eta$ meson yields can indicate the differences in the relative contributions of quarks and gluons. Neutral meson spectra also serve as an input for

(C) O. KOVALENKO, 2019 the direct photon analysis. Direct photons are defined as all photons that are not coming from the decays of particles. Photons do not interact strongly with the medium. They carry information on the properties of the matter at the space-time point of their emission. The high- $p_{\mathrm{T}}$ part of the direct photon spectrum is dominated by photons created in the hard scattering of the partons of incoming nucleons and can serve as a tool to constrain the models that describe the initial stage of a collision. The low- $p_{\mathrm{T}}$ part of the direct photon spectrum may contain photons from the thermal emission of the hot matter and probes its temperature and the velocity of the collective expansion.

\section{Neutral Meson Measurements}

The ALICE experiment is a general-purpose detector [1]. It consists of 17 separate subdetectors that are dedicated to specific goals. Neutral mesons are reconstructed via the two-photon decay channel. Photons in ALICE can be measured in an Electro-Magnetic Calorimeter (EMCal) [2] and a Photon Spectrometer (PHOS) [3] or by means of the Photon Conversion Method (PCM) based on the reconstruction of photons from $e^{+} e^{-}$pairs that are products of the photon conversion in the material of central barrel detectors [4].

The product the efficiency $\varepsilon$ times the acceptance $A$ for different methods of neutral meson reconstruction is shown in Fig. 1. The EMCal has the highest $A \cdot \varepsilon$

ISSN 2071-0194. Ukr. J. Phys. 2019. Vol. 64, No. 7 
values, as it has a large acceptance and a high probability to measure photons. PHOS has a lower $A \cdot \varepsilon$ factor due to limited acceptance, but it has lower energy threshold for the signal and outperforms EMCal at low $p_{\mathrm{T}}$. It is possible to combine the photons reconstructed with EMCal and PCM to form pairs (PCMEMC method). In this case, the $A \cdot \varepsilon$ factor is approximately 10 times smaller than that of EMCal due to the small conversion probability of a photon. This method makes it possible to extend the measurement up to high $p_{\mathrm{T}}$, because showers from different photons don't merge in a detector. The PCM efficiency is determined by the probability of the photon conversion, its $A \cdot \varepsilon$ factor is the lowest.

\subsection{Transverse momentum spectra of neutral mesons in pp collisions}

The ALICE experiment has measured $\pi^{0}$ and $\eta$ meson spectra in pp collisions at several collision energies: $\sqrt{s}=0.9,2.76,7,8 \mathrm{TeV}$ [5-9], see Fig. 2. Neutral pion spectra were reconstructed up to $p_{\mathrm{T}} \sim 40 \mathrm{GeV} / c$ (for $\sqrt{s}=2.76 \mathrm{TeV}$ ). PYTHIA $8.2[10]$ with Monash 2013 tune describes the data at high $p_{\mathrm{T}}$, but shows a deviation from the data at moderate $p_{\mathrm{T}}$ at the higher energies. The NLO calculations [11-13] predict a $20-60 \%$ higher yield, and the difference increases with $p_{\mathrm{T}}$. The situation with $\eta$ meson is similar: PYTHIA 8.2 with Monash 2013 tune reproduces the data, whereas the NLO calculations predict a 50 $100 \%$ higher yield at all colliding energies.

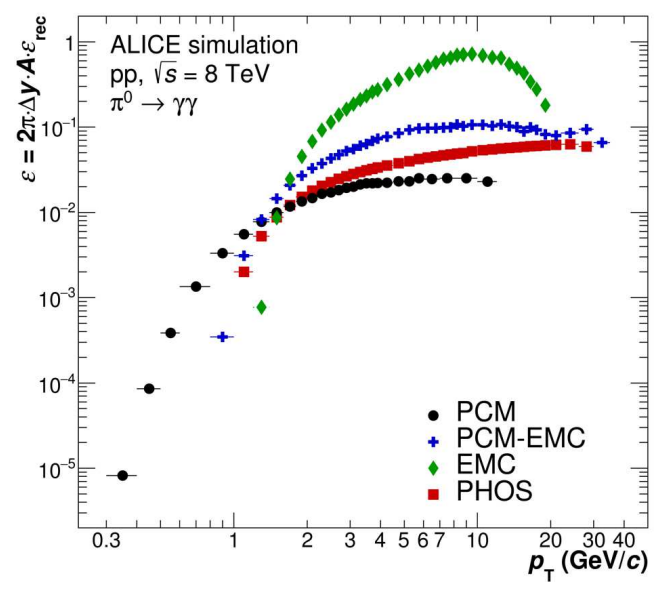

Fig. 1. Normalized correction factors $\epsilon$ for each reconstruction method for $\pi^{0}$ as a function of $p_{\mathrm{T}}$. The factors contain the reconstruction efficiencies and the detector acceptances normalized per unit rapidity and full azimuthal angle

\subsection{Transverse momentum} spectra of neutral mesons in $\mathbf{p - P b}$ collisions

ALICE has recently measured the $\pi^{0}$ and $\eta$ yields in $\mathrm{p}-\mathrm{Pb}$ collisions at $\sqrt{s_{\mathrm{NN}}}=5.02 \mathrm{TeV}$ [15]. Neutral pion and $\eta$ spectra are well described by the Tsallis fits [16]. The NLO pQCD calculations $[11,17]$ scaled

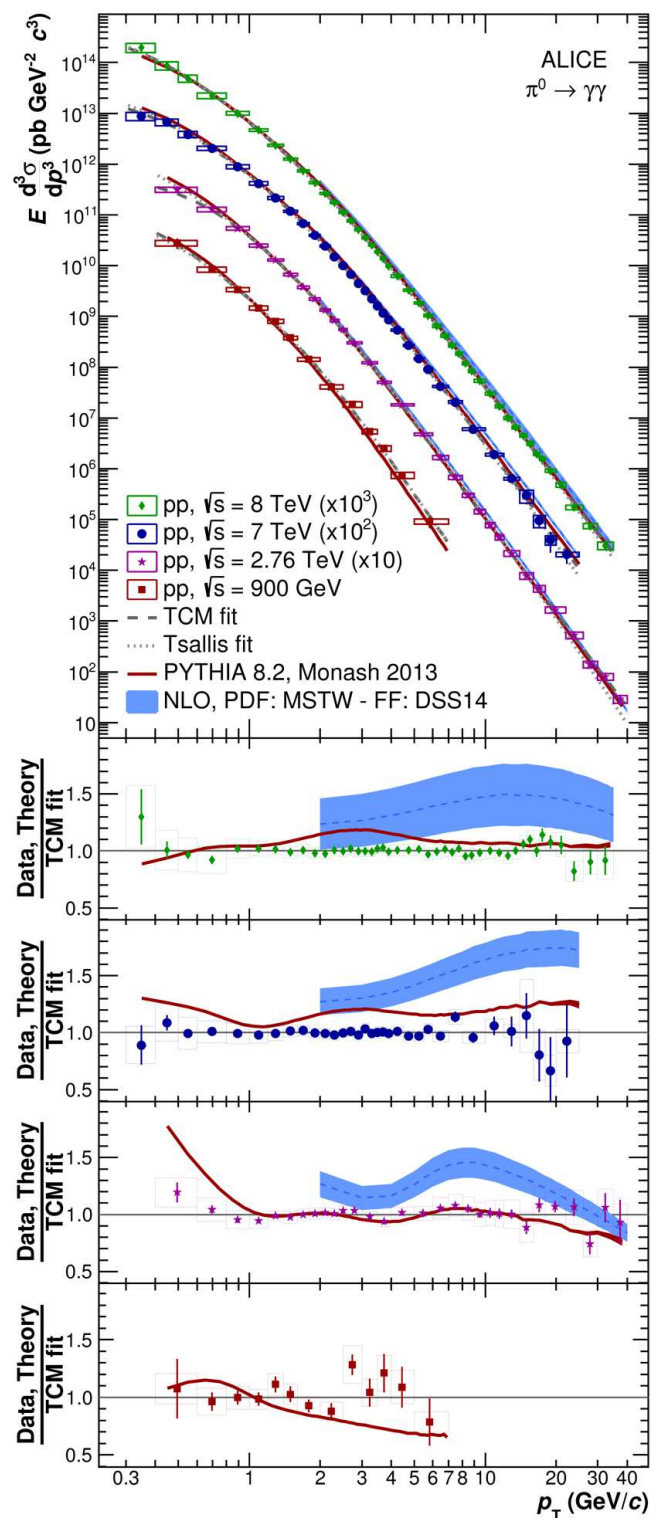

Fig. 2. Neutral pion spectrum at $\sqrt{s}=0.9,2.76,7$, and $8 \mathrm{TeV}$ [5-9]. The spectrum is compared to the PYTHIA8 [10] event generator and NLO pQCD calculations. The ratios of data and predictions to the two-component model (TCM) fit [14] are shown on the bottom panels for each energy separately 


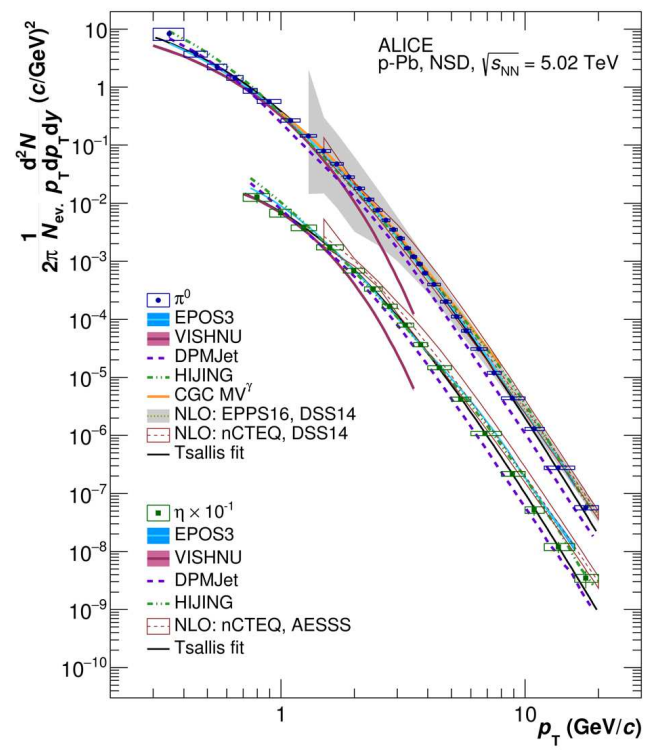

Fig. 3. Neutral pion and $\eta$ spectra measured in p-Pb collisions at $\sqrt{s_{\mathrm{NN}}}=5.02 \mathrm{TeV}[15]$. The data are compared to the scaled NLO pQCD calculations [11,17] and to DPMJET [19], VISHNU [20], HIJING [21], EPOS [18], CGC [22] models

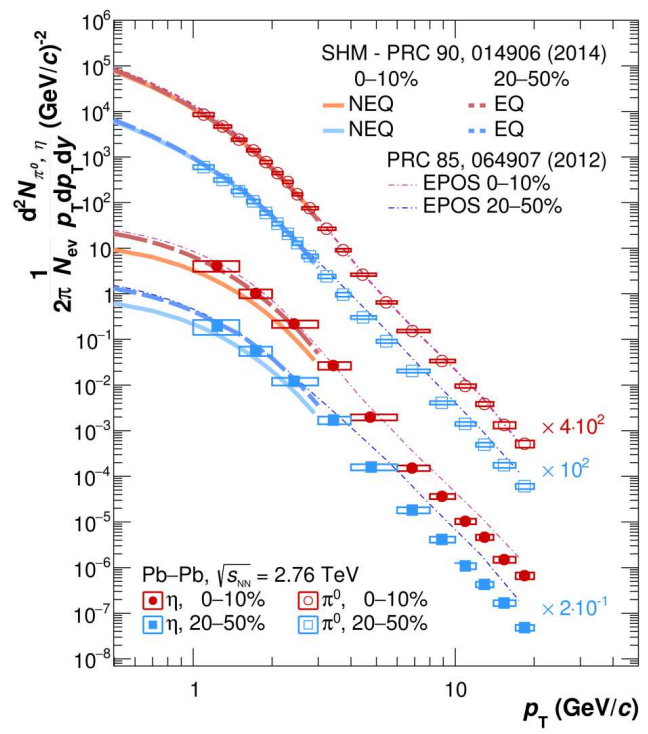

Fig. 4. Neutral pion and $\eta$ spectra in two centrality classes measured in $\mathrm{Pb}-\mathrm{Pb}$ collisions at $\sqrt{s_{\mathrm{NN}}}=2.76 \mathrm{TeV}[6]$

with the number of binary nucleon-nucleon collisions reproduce the $\pi^{0}$ spectrum in the entire $p_{\mathrm{T}}$ range and overpredict the $\eta$ spectrum at high $p_{\mathrm{T}}$. EPOS [18] Monte-Carlo reproduces the $\pi^{0}$ spectrum and $\eta$ spectrum below $3 \mathrm{GeV} / c$, but overpredicts it at high
$p_{\mathrm{T}}$. The hydrodynamic model VISHNU[20] provides a good description at low $p_{\mathrm{T}}$. The HIJING[21] and DPMJET [19] models fail to reproduce data for $p_{\mathrm{T}}$ larger than $4 \mathrm{GeV} / c$.

\subsection{Transverse momentum spectra of neutral mesons in $\mathrm{Pb}-\mathrm{Pb}$ collisions}

The data collected in 2010 allowed the measurement of the spectrum of $\pi^{0}$ in $\mathrm{Pb}-\mathrm{Pb}$ collisions at $\sqrt{s_{\mathrm{NN}}}=2.76 \mathrm{TeV}$ in the range $0.6<p_{\mathrm{T}}<12 \mathrm{GeV} / c$ [6]. The neutral pion yield can be described by the Tsallis fits. Combining the datasets collected in 2010 and 2011 years allowed one to extend the range of the $\pi^{0}$ spectrum up to $20 \mathrm{GeV} / c$ and to measure also the $\eta$ meson spectra in narrower centrality classes [8], see Fig. 4. Two versions of the SHM model [23] reproduce the shape of the $\pi^{0}$ spectrum at low $p_{\mathrm{T}}$. For the $\eta$ mesons, NEQ SHM underestimates the yield at the low- $p_{\mathrm{T}}$ region.

\subsection{Nuclear modification factor in $\mathrm{Pb}-\mathrm{Pb}$ collisions}

Figure 5 shows the nuclear modification factor defined as the meson yield in $\mathrm{Pb}-\mathrm{Pb}$ collisions divided by the meson production cross-section in pp collisions at the same energy scaled with the nuclear overlap function. The value of $R_{\mathrm{AA}}=1$ corresponds to the absence of medium effects. For $\mathrm{Pb}-\mathrm{Pb}$ collisions at $\sqrt{s_{\mathrm{NN}}}=2.76 \mathrm{TeV}, R_{\mathrm{AA}} \sim 0.1$ at $p_{\mathrm{T}} \sim 7 \mathrm{GeV} / c$ was observed reflecting a strong energy loss by partons in the hot quark-gluon matter. The $R_{\mathrm{AA}}$ increases with $p_{\mathrm{T}}$. The nuclear modification factors for $\pi^{0}$ and $\eta$ agree with those for $\pi^{ \pm}$and $K^{ \pm}$. The right plot of Fig. 5 shows the centrality dependence of the nuclear modification factor in $\mathrm{Pb}-\mathrm{Pb}$ collisions. The $R_{\mathrm{AA}}$ decreases, as the centrality increases, indicating that the medium effects are most prominent in the central collisions.

\section{Direct Photons Measurements}

Direct photons are all photons that do not originate from the hadron decays. The yield can be calculated as

$\gamma_{\text {direct }}=\gamma_{\text {inc }}-\gamma_{\text {decay }}=\left(1-1 / R_{\gamma}\right) \gamma_{\text {inc }}$,

where $\gamma_{\text {inc }}-$ the inclusive photon spectrum, $\gamma_{\text {decay }}-$ the decay photon spectrum, $\gamma_{\text {direct }}$ - the direct photon spectrum, and $R_{\gamma}=\gamma_{\text {inc }} / \gamma_{\text {decay }}$. It turns out that the ratio $R_{\gamma}$ expressed as a double ratio $R_{\gamma}=$ 


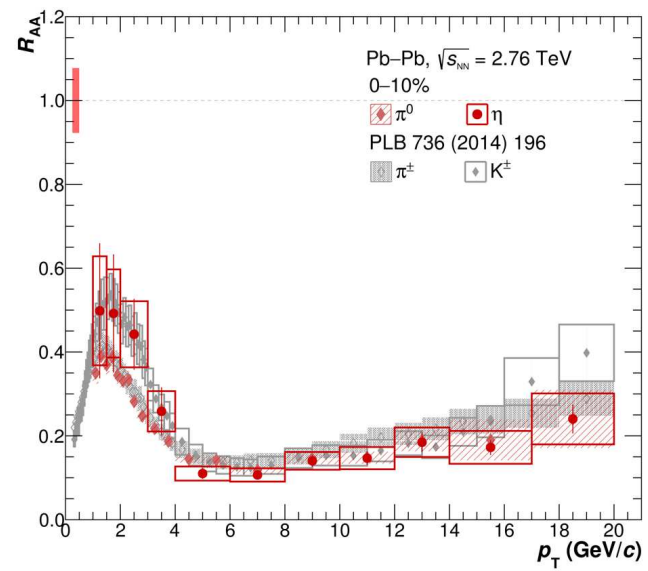

$a$

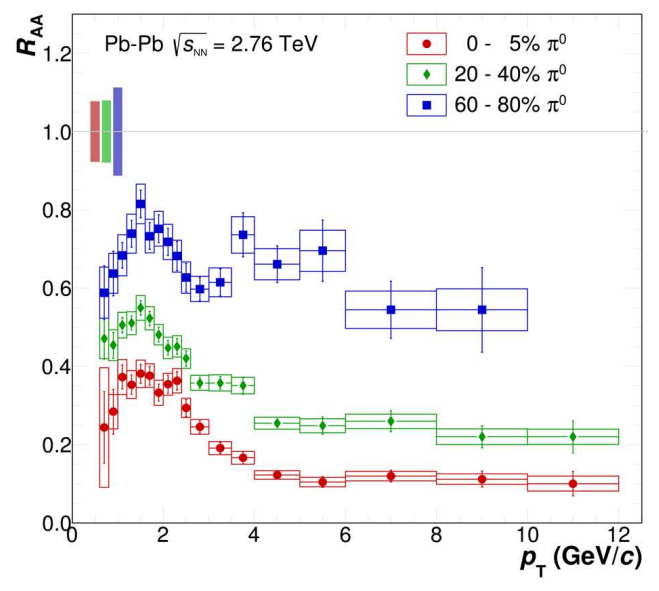

$b$

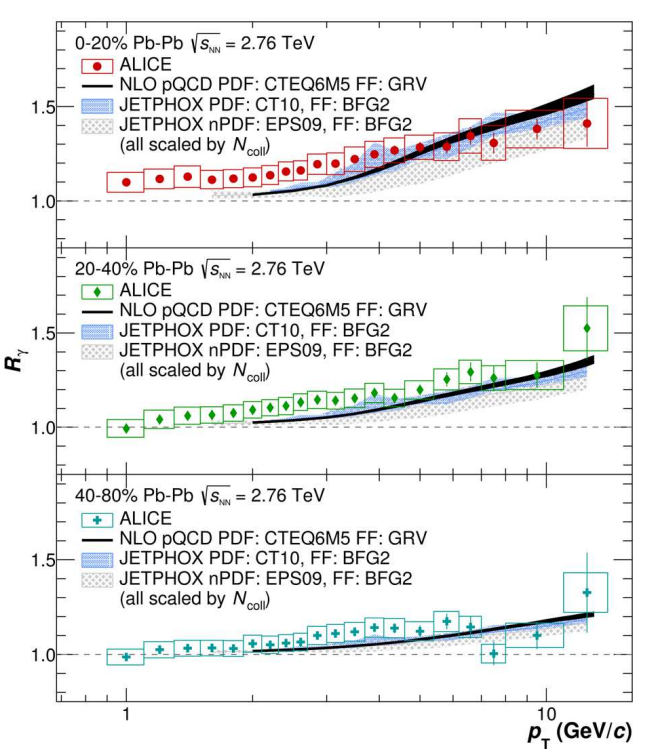

Fig. 6. The double ratio $R_{\gamma}$ measured for three centrality classes in $\mathrm{Pb}-\mathrm{Pb}$ collisions at $\sqrt{s_{\mathrm{NN}}}=2.76 \mathrm{TeV}$ [24] compared to NLO pQCD (for the direct photon yield in pp collisions) and JETPHOX [26] predictions with various PDFs and FFs scaled by the number of binary collisions

inverse slope is found to be equal to $304 \pm 11^{\text {stat }} \pm$ $\pm 40^{\text {sys }} \mathrm{MeV}$. To convert the slope value to the temperature, however, one has to take the expansion of the system into account.

\section{Summary}

ALICE has measured the neutral meson spectra in a wide $p_{\mathrm{T}}$ range in pp collisions at $\sqrt{s}=0.9,2.76$, 7 , and $8 \mathrm{TeV}$. The NLO calculations systematically predict a higher yield, especially at the highest collision energies. The neutral meson spectra measured in $\mathrm{Pb}-\mathrm{Pb}$ collisions at $\sqrt{s_{\mathrm{NN}}}=2.76 \mathrm{TeV}$ were used to calculate nuclear modification factors. The nuclear modification factor measured in $\mathrm{Pb}-\mathrm{Pb}$ shows the strong suppression of the $\pi^{0}$ yield related to the parton energy loss in a hot quark-gluon matter. That can be explained by the final-state effect, as $\mathrm{p}-\mathrm{Pb}$ data are consistent with unity, showing the absence of cold nuclear matter effects. The direct photon spectrum and the double ratio $R_{\gamma}$ were measured in $\mathrm{Pb}-\mathrm{Pb}$ collisions at $\sqrt{s_{\mathrm{NN}}}=2.76 \mathrm{TeV}$ in three centrality classes. The double ratio $R_{\gamma}$ in central $\mathrm{Pb}-\mathrm{Pb}$ collisions exceeds the prompt photon pQCD predictions at $p_{\mathrm{T}}<4 \mathrm{GeV} / c$. The inverse slope of the direct photon spectrum in central $\mathrm{Pb}-\mathrm{Pb}$ collisions is estimated to be $304 \pm 11^{\text {stat }} \pm 40^{\text {sys }} \mathrm{MeV}$. 
1. ALICE Collaboration. The ALICE experiment at the CERN LHC. J. Instrum. 3, S08002 (2008).

2. ALICE Collaboration. ALICE electromagnetic calorimeter: addendum to the ALICE technical proposal. CERNLHCC-2006-014, CERN-LHCC-96-32-ADD-3 (2006).

3. ALICE Collaboration. Technical Design Report of the Photon Spectrometer (PHOS). CERN-LHCC-99-04 (1999).

4. J. Alme et al. The ALICE TPC, a large 3-dimensional tracking device with fast readout for ultra-high multiplicity events. Nucl. Instrum. Meth. A 622, 316 (2010).

5. ALICE Collaboration. Neutral pion and $\eta$ meson production in proton-proton collisions at $\sqrt{s}=0.9 \mathrm{TeV}$ and $\sqrt{s}=7 \mathrm{TeV}$. Phys. Lett. B 717, 162 (2012).

6. ALICE Collaboration. Neutral pion production at midrapidity in pp and $\mathrm{PbPb}$ collisions at $\sqrt{s_{\mathrm{NN}}}=2.76 \mathrm{TeV}$. Eur. Phys. J. C 74, 3108 (2014).

7. ALICE Collaboration. Production of $\pi^{0}$ and $\eta$ mesons up to high transverse momentum in pp collisions at $2.76 \mathrm{TeV}$. Eur. Phys. J. C 77, 339 (2017).

8. ALICE Collaboration. Neutral pion and $\eta$ meson production at mid-rapidity in $\mathrm{Pb}-\mathrm{Pb}$ collisions at $\sqrt{s_{\mathrm{NN}}}=2.76 \mathrm{TeV}$. Phys. Rev. C 98, 044901 (2018).

9. ALICE Collaboration. $\pi^{0}$ and $\eta$ meson production in proton-proton collisions at $\sqrt{s}=8 \mathrm{TeV}$. Eur. Phys. J. C 78, 263 (2018).

10. P. Skands, S. Carrazza, J. Rojo. Tuning PYTHIA 8.1: the Monash 2013 tune. Eur. Phys. J. C74, 3024 (2014).

11. D. de Florian, R. Sassot, M. Epele, R.J. Hernández-Pinto, M. Stratmann. Parton-to-pion fragmentation reloaded. Phys. Rev. D 91, 014035 (2015).

12. W. Tung, H. Lai, A. Belyaev, J. Pumplin, D. Stump, C.-P. Yuan. Heavy quark mass effects in deep inelastic scattering and global QCD analysis. JHEP 02, 053 (2007).

13. C.A. Aidala, F. Ellinghaus, R. Sassot, J.P. Seele, M. Stratmann. Global analysis of fragmentation functions for eta mesons. Phys. Rev. D 83, 034002 (2011).

14. A. Bylinkin, N.S. Chernyavskaya, A.A. Rostovtsev. Predictions on the transverse momentum spectra for charged particle production at LHC-energies from a two component model. Eur. Phys. J. C 75, 166 (2015).

15. ALICE Collaboration. Neutral pion and $\eta$ meson production in p-Pb collisions at $\sqrt{s_{\mathrm{NN}}}=5.02 \mathrm{TeV}$. Eur. Phys. J. C 78, 624 (2018).

16. C. Tsallis. Possible generalization of Boltzmann-Gibbs statistics. J. Statist. Phys. 52479 (1988).

17. B. Jäger, A. Schäfer, M. Stratmann, W. Vogelsang. Nextto-leading order QCD corrections to high- $p_{\mathrm{T}}$ pion production in longitudinally polarized pp collisions. Phys. Rev. D 67, 054005 (2003).

18. K. Werner, B. Guiot, Iu. Karpenko, T. Pierog. Analyzing radial flow features in $\mathrm{p}-\mathrm{Pb}$ and $\mathrm{pp}$ collisions at several $\mathrm{TeV}$ by studying identified-particle production with the event generator EPOS3. Phys. Rev. C 89, 064903 (2014).

19. S. Roesler, R. Engel, J. Ranft. The Monte Carlo Event Generator DPMJET-III. arXiv:0012252 [hep-ph](2010).

20. Chun Shen, Jean-François Paquet, Gabriel S. Denicol Sangyong Jeon, Charles Gale. Collectivity and electromagnetic radiation in small systems. Phys. Rev. C 95, 014906 (2017).

21. M. Gyulassy, X.N. Wang. HIJING 1.0: A Monte Carlo program for parton and particle production in high energy hadronic and nuclear collisions. Comput. Phys. Commun. 83, 307 (1994).

22. T. Lappi, H. Mäntysaari. Single inclusive particle production at high energy from HERA data to proton-nucleus collisions. Phys. Rev. D 88, 114020 (2013).

23. V. Begun, W. Florkowski, M. Rybczynski. Explanation of hadron transverse-momentum spectra in heavy-ion collisions at $\sqrt{s_{\mathrm{NN}}}=2.76 \mathrm{TeV}$ within a chemical nonequilibrium statistical hadronization model. Phys. Rev. C 90, 014906 (2014).

24. ALICE Collaboration. Direct photon production in $\mathrm{Pb}-\mathrm{Pb}$ collisions at $\sqrt{s_{\mathrm{NN}}}=2.76 \mathrm{TeV}$. Phys. Lett. $B$ 235-248s, 754 (2019).

25. S. Catani, M. Fontannaz, J.P. Guillet, E. Pilon. Cross section of isolated prompt photons in hadron-hadron collisions. JHEP 0205, 028 (2002).

26. P. Aurenche, M. Fontannaz, J.P. Guillet, E. Pilon, M. Werlen. Recent critical study of photon production in hadronic collisions. Phys. Rev. D 73, 094007 (2006).

Received 08.07.19

О. Коваленко, від імені Колабораціӥ АLICE

ВИМІРЮВАННЯ НЕЙТРАЛЬНИХ МЕЗОНІВ

ТА ПРЯМИХ ФОТОНІВ В ЕКСПЕРИМЕНТАХ ALICE

Р е $з$ ю м е

Експеримент ALICE заплановано для вивчення властивостей речовини, що народжується в зіткненнях протонів та важких іонів на LHC. Нейтральні мезони можна відтворити в ALICE в широкому інтервалі поперечних імпульсів за допомогою двофотонних розпадів. Вимірювання нейтральних мезонів у зіткненнях протонів дають можливість перевірити пертурбативну KXД в NLO та NNLO наближеннях, а також уточнити функції розподілу та фрагментації партонів. Спектри нейтральних мезонів, виміряних у $p \mathrm{~A}$ та АА зіткненнях, дозволяють перевірити модифікацію партонної функції розподілу в ядрі і втрату енергії партонів у гарячій речовині, що утворюється в АА зіткненнях. Нами представлено останні результати ALICE стосовно вимірювання прямих фотонів у $\mathrm{Pb}-\mathrm{Pb}$ зіткненнях, продукування нейтральних піонів та $\eta$ мезонів у зіткненнях $p p, p-\mathrm{Pb}$ та $\mathrm{Pb}-\mathrm{Pb}$. 